\title{
Communicable Diseases Report, NSW, January and February 2009
}

\section{Communicable Diseases Branch NSW Department of Health}

For updated information, including data and facts on specific diseases, visit www.health.nsw.gov.au and click on Infectious Diseases or access the site directly at: http://www.health.nsw.gov.au/ publichealth/infectious/index.asp.

Figure 1 and Tables 1 and 2 show reports of communicable diseases received through to the end of February 2009 in New South Wales (NSW).

\section{Vaccine-preventable diseases \\ Pertussis (whooping cough)}

Pertussis notifications continue to increase with more than 3900 cases reported in NSW in January and February. While babies and young children represent the largest proportion of notifications, an increase has also been seen in older children and adults. Increased use of polymerase chain reaction (PCR), a diagnostic test for pertussis, may account for some of the increase. Many cases have good evidence of past pertussis immunisation: the vaccine provides some protection for several years; however, pertussis immunity typically wanes thereafter.

\section{Measles}

Five confirmed cases of measles were notified in NSW in January and February and another case is under investigation. Four cases had recently returned from overseas (Thailand, the United States of America and Vietnam). The case who returned from Vietnam was identified through contact tracing of a case on the same flight reported from another jurisdiction. As Vietnam is currently experiencing an outbreak of measles, it is difficult to determine whether transmission occurred during the flight or prior to boarding. The most recent case appears to have acquired measles locally, although a source case can not be identified.

\section{Invasive meningococcal disease}

Twelve cases of invasive meningococcal disease were reported up to the end of February 2009 in NSW, compared with five cases in the same period in 2008. Of the 12 cases, nine were serogroup $\mathrm{B}$, one was of unknown serogroup and two cases were serogroup $C$ (the strain prevented by the current vaccine). Overall, rates of meningococcal disease have continued to decline since 2000 .

\section{Mumps}

Three cases of mumps have been reported in NSW so far this year. The outbreak seen in young adults mainly around south-eastern and northern Sydney in 2008 appears to have passed.

\section{Rubella}

One case of rubella has been reported in NSW so far this year in a man who had recently travelled overseas.

\section{Tetanus}

One case of tetanus has been reported in NSW so far this year in a man who presented with tetanus after a penetrating foot injury. The diagnosis was made clinically.

\section{Influenza}

Influenza outbreaks occur each winter in NSW. The influenza virus mutates frequently and new strains emerge regularly. Vaccination is the mainstay of influenza prevention and provides good protection when the strain circulating matches that in the vaccine. Vaccine manufacturers review strains that were circulating internationally several months before winter and typically include three strains of killed influenza in the vaccine. It is impossible to predict in advance the severity of the coming influenza season, as it is largely dependent on the degree of mutation, if any, of circulating influenza strains.

The anti-influenza drugs, oseltamivir and zanamivir, can reduce the severity of illness if given early in the course of infection and can prevent illness in people exposed to an infectious person. These drugs must be prescribed by a doctor and to date have not been widely used in Australia. Analysis of strains of influenza virus in 2008 has found that one strain, influenza $\mathrm{A} \mathrm{H1N1}$, is highly resistant to oseltamivir. ${ }^{1}$ In the past, this strain has been relatively uncommon in Australia, but it is included in this year's vaccine. 
In preparation for the 2009 influenza season, NSW Health has:

- faxed general practitioners and Aboriginal Medical Services with order forms for free influenza vaccine for some people at high risk (those aged over 65 and Aboriginal people aged over 50 or between 15 and 49 years with underlying illnesses);

- written to aged-care facilities promoting vaccine use in residents (provided free) and staff; and

- continued to provide free vaccine for use by area health services for health-care workers.

\section{Enteric diseases \\ Cryptosporidiosis}

In February 2009, an increase in cryptosporidiosis cases was identified across NSW. Cryptosporidiosis is a disease caused by infection with the parasite Cryptosporidium. Infection causes diarrhoea and abdominal cramps that can last for many weeks. There is no specific treatment. The disease is spread in several ways, most importantly by direct contact with people or animals with the infection, or by drinking contaminated water, including inadvertently while swimming. Large outbreaks have occurred in NSW in previous years and were linked to contaminated swimming pools. In the current outbreak, many cases reported swimming as a risk factor, but in a range of different pools.

There is normally a small risk of acquiring cryptosporidiosis from ingesting water while swimming in pools. This risk is likely to be higher now given the number of cases being notified in the general community, but can be reduced by not swallowing water from the pool. There is no public health recommendation to avoid swimming in public pools for well people; however, people with diarrhoea in the previous 2 weeks should not enter pools or spas, and all swimmers should follow good hygiene practices. Person-to-person transmission can be reduced by regular hand-washing with soap and running water for 10 seconds.

\section{Shigellosis}

Shigella infections remain elevated in men in eastern Sydney, possibly related to male-to-male sexual activities. Careful attention to hygiene (hand-washing with soap and water for at least 10 seconds) after using the toilet, before handling food, and before and after sex, will help reduce spread. People with diarrhoea should not handle food for others and avoid direct contact with others until at least 48 hours after symptoms resolve. Antibiotic treatment is recommended for patients with Shigella infection to reduce the likelihood of transmitting the infection to others.

\section{Gastroenteritis}

In January and February 2009, NSW public health units investigated 48 outbreaks of gastroenteritis, including 43 suspected to be caused by person-to-person transmission, and five suspected to be the result of foodborne transmission.

The 43 suspected person-to-person outbreaks affected a total of 475 people. Twenty-six occurred in aged-care facilities and affected 332 people; five occurred in hospitals and affected 52 people; nine occurred in child-care centres and affected 81 people; and three outbreaks in other institutions affected 10 people. Clinical specimens were submitted for testing from 16 suspected personto-person gastroenteritis outbreaks. Norovirus was confirmed in stool samples from six outbreaks in aged-care facilities. The causative agent was not determined for the remaining outbreaks.

Of the five suspected foodborne outbreaks, four were outbreaks of salmonellosis affecting 127 people, of whom 43 had laboratory confirmation of the diagnosis. One outbreak affecting six people was likely to be caused by a bacterial toxin. Deficiencies in food handling were the likely cause of all outbreaks.

\section{Sexually transmissible infections \\ Syphilis}

Syphilis remains a concern in inner Sydney, mainly among men who have sex with men. Safe sex and regular screening for those with multiple partners are important preventive measures.

\section{Vectorborne diseases \\ Dengue}

Forty-eight cases of dengue fever were reported in NSW residents in January and February. Of these, three acquired the infection in Cairns and the remainder acquired the infection overseas. On the Australian mainland, dengue is transmitted by the Aedes aegypti mosquito, a species not currently found in NSW. There are ongoing outbreaks of dengue fever in Cairns, Townsville, Port Douglas, Yarrabah, Injinoo and Innisfail.

\section{Reference}

1. Centers for Disease Control and Prevention. [Health Alert Network Message on the Internet]. CDC Issues Interim Recommendations for the Use of Influenza Antiviral Medications in the Setting of Oseltamivir Resistance among Circulating Influenza A (H1N1) Viruses, 2008-09 Influenza Season [updated 19 December 2008]. Available at: http://www2a.cdc.gov/HAN/ArchiveSys/ViewMsgV.asp?Alert Num=00279 (Cited 8 March 2009.) 
Figure 1. Reports of selected communicable diseases, NSW, January 2004 to February 2009, by month of onset. Preliminary data: case counts in recent months may increase because of reporting delays. Laboratory-confirmed cases only, except for measles, meningococcal disease and pertussis. BFV, Barmah Forest virus infection; RRV, Ross River virus infection; lab conf, laboratory confirmed; Men $\mathrm{Gp} C$ and $\mathrm{Gp} \mathrm{B}$, meningococcal disease due to serogroup $C$ and serogroup B infection; other/unk, other or unknown serogroups.

NB: Multiple series in graphs are stacked, except gastroenteritis outbreaks.

NB: Outbreaks are more likely to be reported by nursing homes and hospitals than by other institutions.

\begin{tabular}{|lr|}
\hline NSW Population \\
Male & $50 \%$ \\
$<5$ y & $7 \%$ \\
$5-24$ y & $27 \%$ \\
$25-64$ y & $53 \%$ \\
$65+$ y & $13 \%$ \\
Rural & $46 \%$ \\
\hline
\end{tabular}
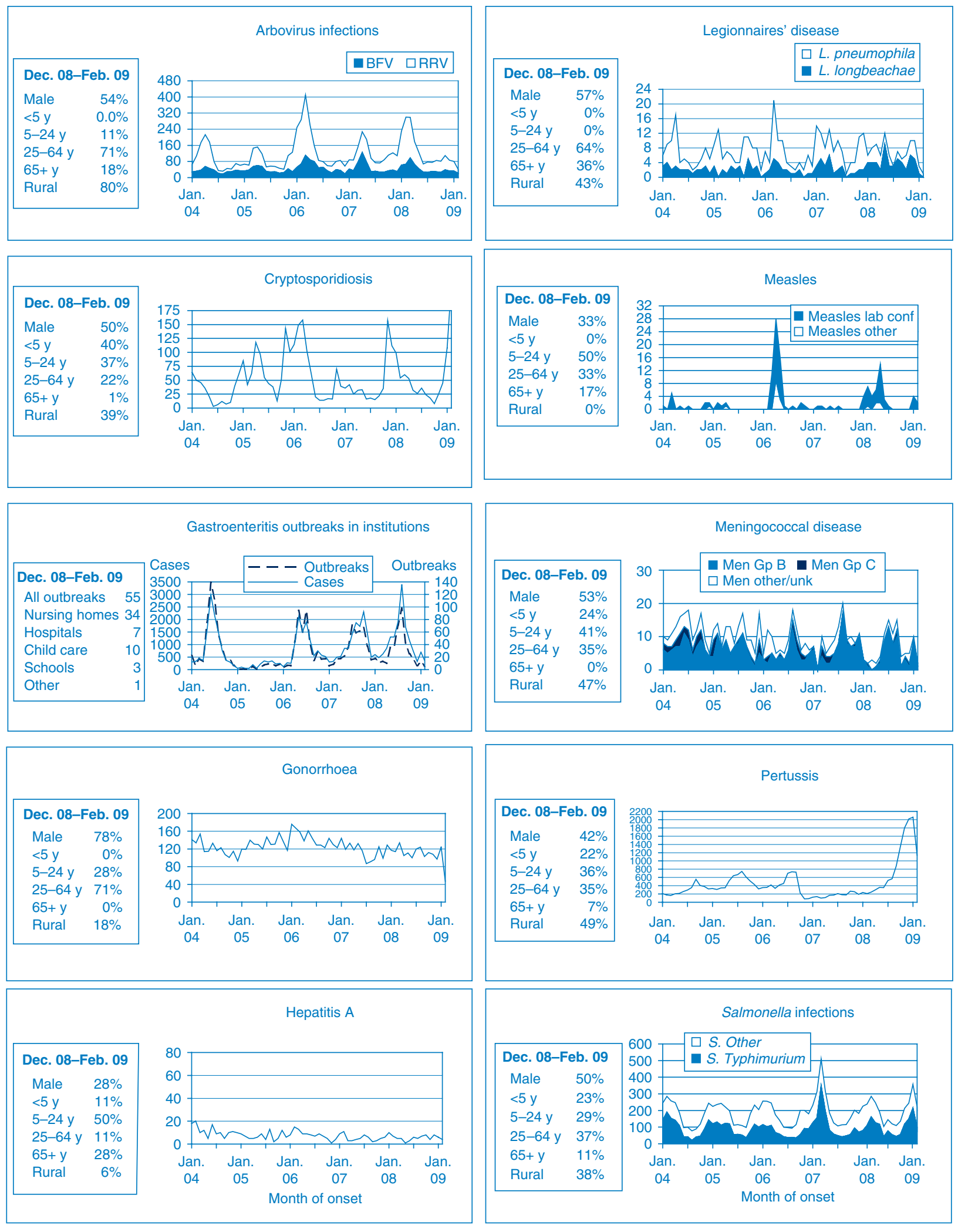


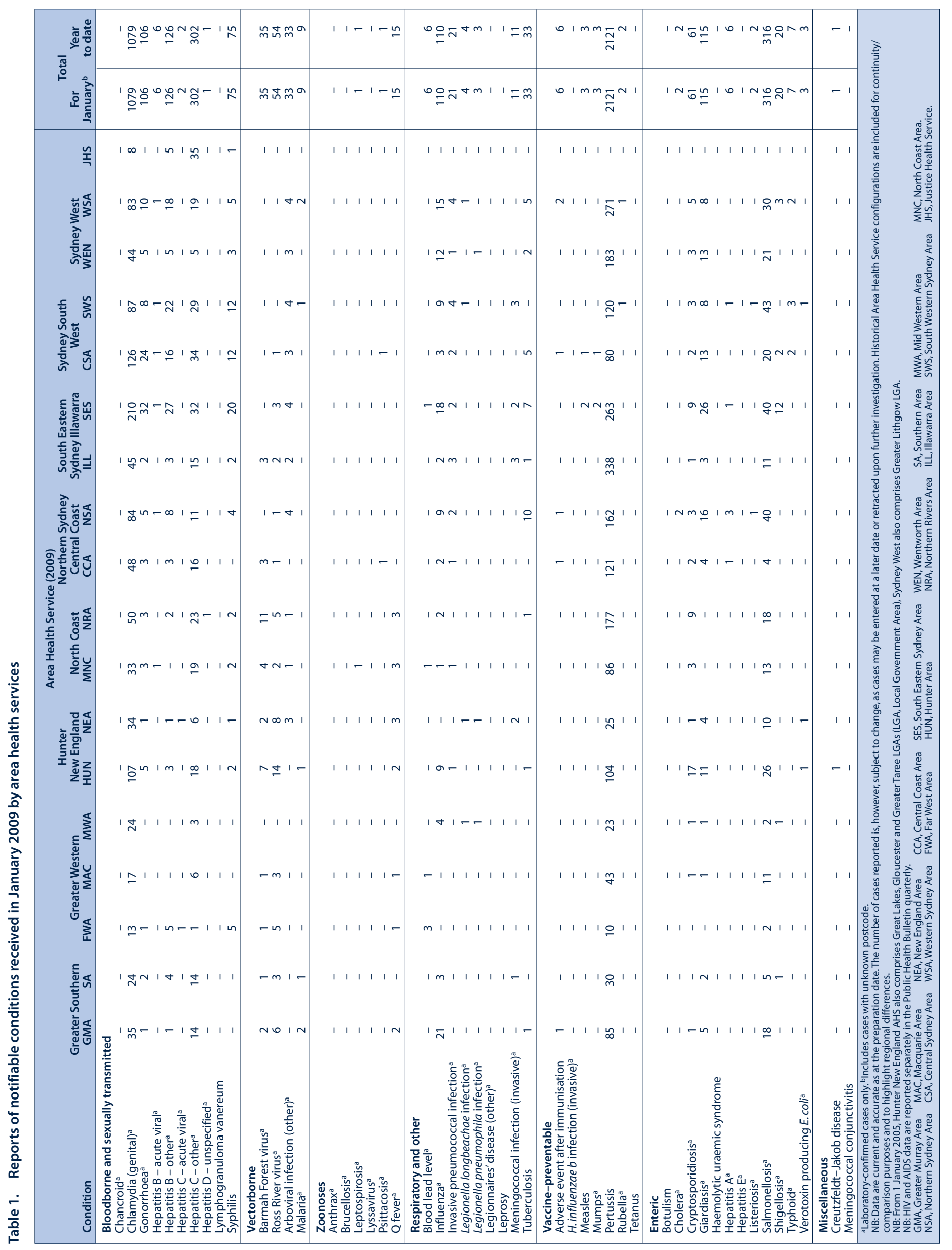




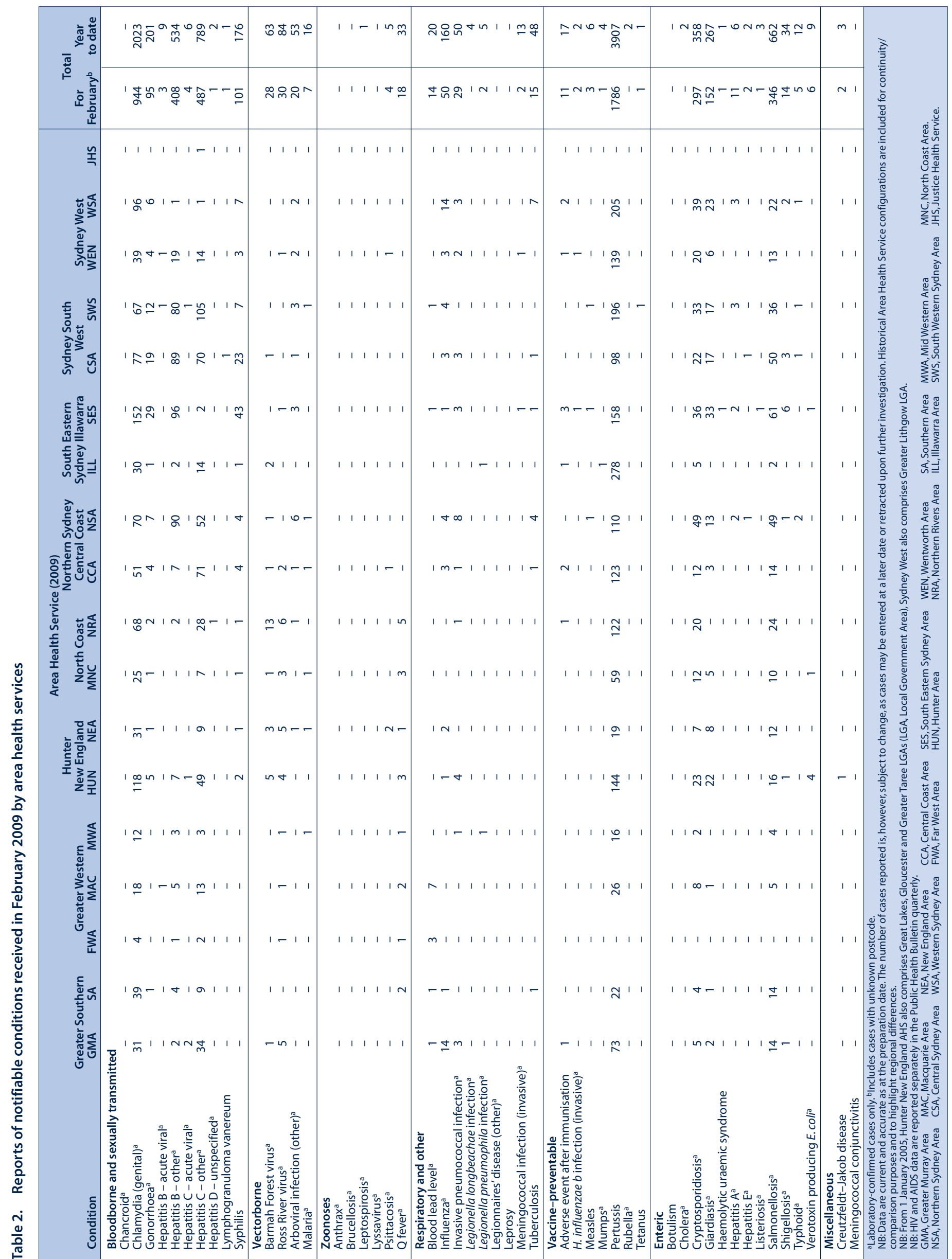

